\title{
Novel tubulin-polymerization inhibitor derived from thalidomide directly induces apoptosis in human multiple myeloma cells: Possible anti-myeloma mechanism of thalidomide
}

\author{
TOYOTAKA IGUCHI ${ }^{1}$, TOMOMI YACHIDE-NOGUCHI ${ }^{2}$, YUICHI HASHIMOTO ${ }^{2}$, \\ SAWAKO NAKAZATO ${ }^{1}$, MORIHIKO SAGAWA ${ }^{1}$, YASUO IKEDA ${ }^{1}$ and MASAHIRO KIZAKI ${ }^{1,3}$ \\ ${ }^{1}$ Division of Hematology, Department of Internal Medicine, Keio University School of Medicine, Tokyo; \\ ${ }^{2}$ Laboratory of Bioorganic Chemistry, Institute of Molecular and Cellular Biosciences, The University of Tokyo, Tokyo; \\ ${ }^{3}$ Division of Hematology, Department of Internal Medicine, Saitama Medical Center, \\ Saitama Medical University, Saitama, Japan
}

Received August 31, 2007; Accepted October 16, 2007

\begin{abstract}
To ascertain the exact anti-myeloma mechanism of thalidomide in vivo, we performed structural development studies of thalidomide, and obtained various analogues with specific molecular properties. Among these derivatives, we found that a new thalidomide analogue, 2-(2,6-diisopropylphenyl)-5-hydroxy- $1 \mathrm{H}$-isoindole-1,3-dione (5HPP-33) had the most potent anti-myeloma effect and tubulin-polymerization-inhibiting activity. 5HPP-33 directly inhibited the growth and survival of various myeloma cell lines (RPMI8226, U266, and IM9) in a dose-dependent manner with $\mathrm{IC}_{50}$ of $1-10 \mu \mathrm{M}$. In contrast, thalidomide itself did not inhibit cellular growth of RPMI8226 cells. Cultivation with $10 \mu \mathrm{M} 5 \mathrm{HPP}-33$ induced $\mathrm{G} 2 / \mathrm{M}$ phase cell cycle arrest, followed by apoptosis of myeloma cells. Treatment with 5HPP-33 induced caspase-3 activity and PARP cleavage. A tubulin polymerization assay using microtubule protein from porcine brain revealed that 5HPP-33 showed potent tubulinpolymerization-inhibiting activity with $\mathrm{IC}_{50}$ of $8.1 \mu \mathrm{M}$, comparable to that of the known tubulin-polymerization inhibitor, rhizoxin. Moreover, its activity was more potent than that of a known thalidomide metabolite, 5-hydroxythalidomide. Notably, the structural requirement for its activity was critical, as other analogues and derivatives of
\end{abstract}

Correspondence to: Dr Masahiro Kizaki, Division of Hematology, Department of Internal Medicine, Saitama Medical Center, Saitama Medical University, 1981 Tsujido-machi, Kamoda, Kawagoe 350-8550, Japan

E-mail: makizaki@saitama-med.ac.jp

Key words: 2-(2,6-diisopropylphenyl)-5-hydroxy-1H-isoindole1,3-dione, tubulin-polymerization inhibitor, thalidomide, multiple myeloma
5HPP-33 showed only slight tubulin-polymerizationinhibiting activity. Our data suggest that $5 \mathrm{HPP}-33$ is a promising candidates for a therapeutic agent of multiple myeloma. In addition, these results suggest that the tubulinpolymerization inhibiting activity of thalidomide might be a possible mechanism for inducing the apoptosis of myeloma cells by thalidomide.

\section{Introduction}

Multiple myeloma is characterized by the proliferation of plasma cells that produce monoclonal immunoglobulin. Despite the conventional use of high-dose chemotherapy with hematopoietic stem cell transplantation, the disease eventually reappears resulting in an incurable hematological malignancy $(1,2)$. Therefore, novel therapeutic approaches are desirable in clinical settings. Recently, thalidomide has been introduced in the treatment of myeloma, and many clinical trials have since confirmed its efficiency in patients with recurring, refractory or newly diagnosed multiple myeloma (3-5). Multiple mechanisms have been proposed to explain the anti-myeloma activity of thalidomide $(1,6,7)$. However, its precise mechanism of action is still unclear, as thalidomide rapidly undergoes spontaneous, nonenzymatic, hydrolytic cleavage to numerous metabolites in vivo (4). In addition, the side effects of thalidomide and its teratogenic potential have often prevented its use in the direct treatment of myeloma (4). To address the exact anti-myeloma effect of thalidomide and to develop new derivatives without causing teratogenic effects, we studied the structural development of thalidomide and obtained various analogues with specific molecular properties $(8,9)$. We found that, among these derivatives, 2-(2,6-diisopropylphenyl)-5-hydroxy- $1 H$ isoindole-1,3-dione (5HPP-33) had the most potent antimyeloma effect and tubulin-polymerization-inhibiting activity $(10,11)$. The results provide us with the framework for future clinical studies of 5HPP-33 to improve patient outcome in multiple myeloma, and suggest a new mechanism of thalidomide against myeloma cells. 


\section{Materials and methods}

Cells and culture condition. The human multiple myeloma cell lines, RPMI8226, IM9 and U266, were obtained from the Japan Cancer Research Resources Bank (Tokyo, Japan), and were cultured in RPMI-1640 medium (Gibco-BRL, Grand Island, NY) with $10 \%$ heat-inactivated fetal bovine serum (FBS) (Life Technologies, Rockville, MD), 100 U/ml penicillin $\mathrm{G}$, and $100 \mu \mathrm{g} / \mathrm{ml}$ streptomycin in a humidified atmosphere of $5 \% \mathrm{CO}_{2}$.

Reagents. Our previous reports indicated that the TNF- $\alpha$ production-regulating activity of thalidomide has potent multitarget chemicals $(8,9)$. Therefore, we studied the structural development of thalidomide, and obtained various chemicals, including 5HPP-33. The chemical structure of 5HPP-33 is similar to that of a thalidomide metabolite, 5-hydroxythalidomide (Fig. 1A). Paclitaxel, doxorubicin, and vincristine were purchased from Sigma Chemical (St. Louis, MO). These agents were dissolved in dimethyl sulfoxide (DMSO, Sigma) at concentrations of 1,10 , and $100 \mathrm{mM}$, respectively. The final DMSO concentrations in the medium were not greater than $0.1 \%$.

Assays for cellular proliferation. Cellular proliferation was studied by counting the viable cells and measured using a 3(4,5-dimethylthiazol-2-yl)-5-(3-carboxymethoxyphenyl)-2(4-sulphophenyl)-2H-tetrazolium, inner salt (MTS) proliferation assay kit with CellTiter96 AQueous One Solution Reagent (Promega, Madison, WI). The cells were exposed to MTS for the last $4 \mathrm{~h}$ of culture, and absorbance was measured using a plate reader (Molecular Devices, Sunnyvale, CA) at an optical density (OD) of $440 \mathrm{~nm}$. Viable cells were counted by trypan blue dye exclusion test. For the MTS assay, cells were plated on 96 -well tissue culture plates at $5 \times 10^{4} / \mathrm{ml}$ in a total volume of $100 \mu \mathrm{l}$ with the indicated agents and assayed according to the manufacturer's instructions. The absorbance at $490 \mathrm{~nm}$ was expressed as a relative value of the control culture.

Assays for apoptosis. Apoptosis was determined by morphologic change as well as staining with Annexin V-FITC and propidium iodide (PI) labeling by using a staining kit purchased from BD Bioscience Pharmingen (San Diego, CA). Cells $\left(1 \times 10^{5}\right)$ were incubated with or without $10 \mu \mathrm{M} 5 \mathrm{HPP}-$ 33 for the indicated times followed by cell cycle analysis.

Cell cycle analysis. Cells were suspended in a hypotonic solution $[0.1 \%$ Triton $\mathrm{X}-100,1 \mathrm{mM}$ Tris- $\mathrm{HCl}$ (pH 8.0), $3.4 \mathrm{mM}$ sodium citrate, $0.1 \mathrm{mM}$ EDTA] and stained with $50 \mu \mathrm{g} / \mathrm{ml}$ of PI. The population of cells in each cell cycle phase was determined using ModiFIT software (Becton Dickinson, Franklin Lakes, NJ).

Effects of 5HPP-33 on caspase activity. Activation of caspase- 3 was analyzed using a commercially available caspase- 3 assay kit from Pharmingen. Briefly, FITCconjugated antibody against the active form of caspase- 3 provided in the kit was used for FACS analysis according to the manufacturer's instructions.
Western blot analysis. Cells were collected by centrifugation at $700 \mathrm{x} \mathrm{g}$ for $10 \mathrm{~min}$, and then the pellets were re-suspended in lysis buffer [20 mM Tris- $\mathrm{HCl}(\mathrm{pH} 8.0), 137 \mathrm{mM} \mathrm{NaCl}$, $1.5 \mathrm{mM} \mathrm{MgCl}_{2}, 1 \mathrm{mM}$ ethyleneglycol bis(2-aminoethylether) tetraacetic acid (EGTA), 10\% glycerol, $100 \mathrm{mM} \mathrm{NaF}$, and $1 \%$ Triton X-100]. The protein concentration of lysates was measured by Protein DC Assay kit (Bio-Rad, Hercules, CA). Protein lysate $(20 \mu \mathrm{g})$ was suspended in $2 \mathrm{X}$ gel electrophoresis loading buffer and heated to $90^{\circ} \mathrm{C}$ for $5 \mathrm{~min}$. The lysates were subjected to sodium dodecyl sulfate-polyacrylamide gel electrophoresis (SDS-PAGE) and Western blot analysis. ECL Western blotting detection reagents were used to visualize the target protein (Amersham, Piscataway, $\mathrm{NJ}$ ). The antibodies used were as follows: B-actin (Santa Cruz, CA) and cleaved poly(adenosine diphosphate-ribose) polymerase (PARP), (Cell Signaling Technology, Beverly, MA).

Tubulin-polymerization inhibiting assay. Microtubule protein was prepared from porcine brain as follows: fresh brains were cooled on ice and washed with an aqueous solution containing $100 \mathrm{mM}$ 4-morpholinoethanesulfonic acid (MES), $1 \mathrm{mM}$ EGTA, $1 \mathrm{mM}$ 2-mercaptoethanol (2-ME), and $0.5 \mathrm{mM}$ $\mathrm{MgCl}_{2}$ ( $\mathrm{pH}$ 6.5), and were then homogenized in MES buffer (100 mM MES, 1 mM EGTA, 0.5 mM 2-ME, 1 mM GTP) in a Waring blender under ice. After centrifugation at 50,000 x g for $30 \mathrm{~min}$ at $4^{\circ} \mathrm{C}$, the supernatant was mixed with an equal volume of glycerol buffer (100 mM MES, 1 mM EGTA, $0.5 \mathrm{mM} \mathrm{MgCl} 2,1 \mathrm{mM}$ GTP, $8 \mathrm{M}$ glycerol) and warmed at $37^{\circ} \mathrm{C}$ for $40 \mathrm{~min}$ to polymerize the tubulin. The polymerized tubulin in the solution was collected as a pellet by centrifugation at $100,000 \mathrm{x} \mathrm{g}$ for $45 \mathrm{~min}$ at $25^{\circ} \mathrm{C}$. The pellet was resuspended in $100 \mu 1$ of MES buffer. After homogenization in a Teflon/glass homogenizer, it was chilled at $0^{\circ} \mathrm{C}$ for $30 \mathrm{~min}$. An equal volume of glycerol buffer was added to the supernatant solution, and the solution was stored at $-70^{\circ} \mathrm{C}$. Immediately before use, the protein was further purified from the stock solution by a repeated cycle of the polymerization step. Polymerization of tubulin was followed by turbidity measurement at $37^{\circ} \mathrm{C}$ in an MES buffer solution containing $2 \mathrm{mg}$ of protein per $\mathrm{ml}$ of solution. The turbidity was measured at $400 \mathrm{~nm}$ on a photospectrometer.

Immunofluorescent microscopy. Before cytospinning cells onto slides, RPMI8226 and U266 cells were cultured with or without $10 \mu \mathrm{M} 5 \mathrm{HPP}-33$ for $24 \mathrm{~h}$. Cells were centrifuged at $700 \mathrm{rpm}$ for $5 \mathrm{~min}$ onto glass slides, and placed immediately in $100 \%$ methanol at room temperature for $20 \mathrm{~min}$ to fix them. The slides were washed in PBS three times for $5 \mathrm{~min}$. Anti- $\alpha$-tubulin (Sigma) at $10 \mu \mathrm{g} / \mathrm{ml}$ was added to each slide and incubated for $2 \mathrm{~h}$. The slides were washed in PBS three times for $5 \mathrm{~min}$ at a time, and secondary antibody goat antimouse immunoglobulin, Alexa Fluor 488 (1:200, Molecular Probes, Eugene, OR), was added to the slides for $60 \mathrm{~min}$. The slides were washed in PBS three times for $5 \mathrm{~min}$ at a time and Vectashield (Vector Laboratories, Inc., Burlingame, CA), an anti-fading medium with PI, was mounted on the slides, which were then stored at $4^{\circ} \mathrm{C}$ in the dark. Images of microtubule morphology were captured using a confocal microscope, LSM510 (Carl Zeiss, Wetzlar, Germany). 
A

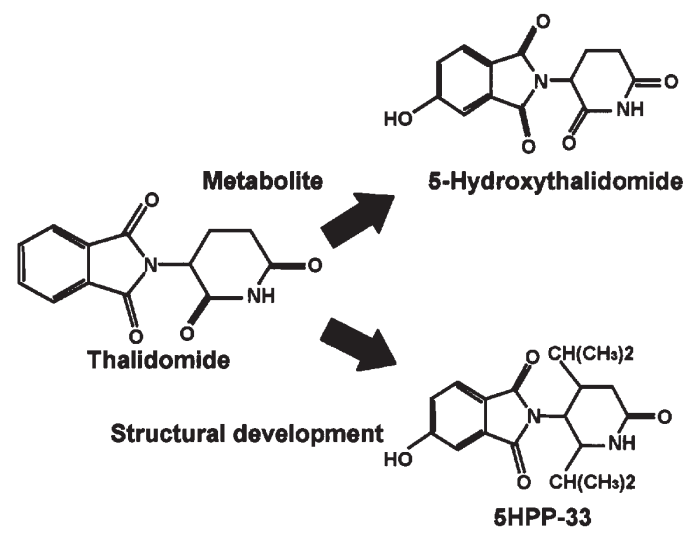

C
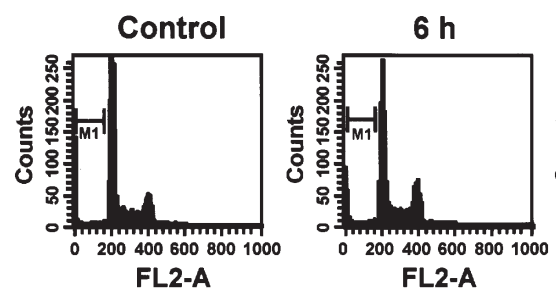

FL2-A

B

Thalidomide

(OD)

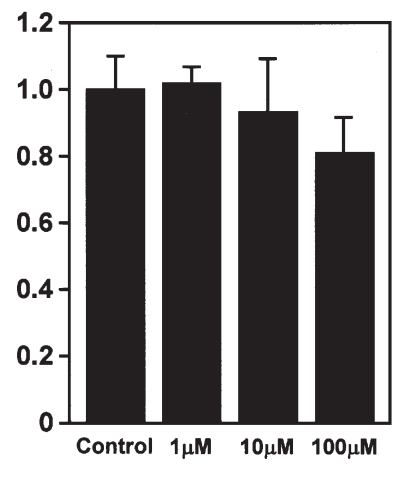

5HPP-33

(OD)

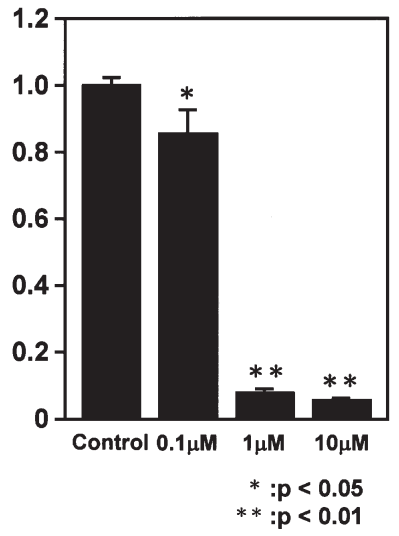

D
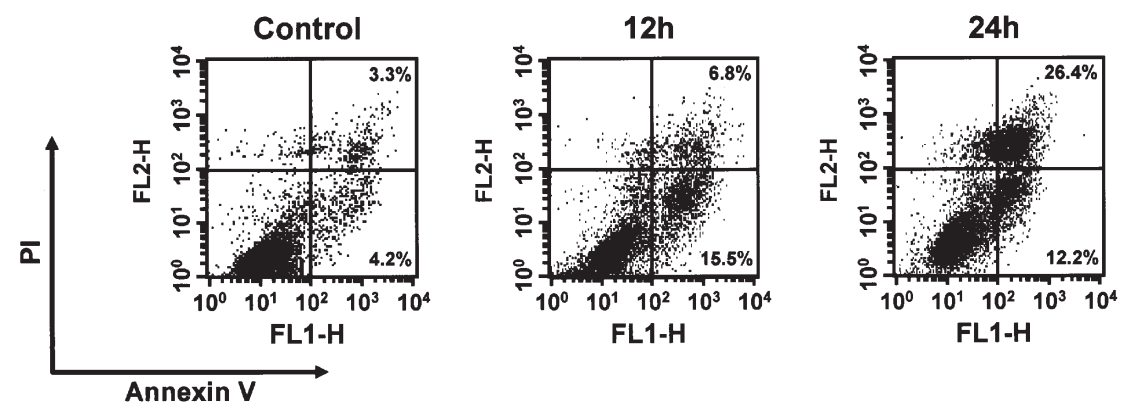

Figure 1. 5HPP-33 induces G2/M cell cycle arrest followed by apoptosis in myeloma cells. (A) Chemical structures of 5HPP-33 and thalidomide metabolite, 5-hydroxythalidomide. (B) Effects of thalidomide and 5HPP-33 on the proliferation of human multiple myeloma cell line RPMI8226. Cells were incubated with indicated concentrations $(0-100 \mu \mathrm{M})$ of thalidomide or 5HPP-33 for $48 \mathrm{~h}$, and then cell proliferation was determined by MTS assay. The assays were performed in triplicate, and bars represent standard deviations. Significant $(* \mathrm{p}<0.05, * \mathrm{*}<0.01)$ differences compared to control. (C) $5 \mathrm{HPP}-33$ induced G2/Mphase cell cycle arrest. RPMI8226 cells were treated with or without $10 \mu \mathrm{M} 5 \mathrm{HPP}-33$ for the indicated times, and then stained with PI as described in Materials and methods. The DNA content was analyzed by means of flow cytometry. (D) Detection of apoptotic cells by Annexin V and PI double staining. RPMI8226 cells were cultured with $10 \mu \mathrm{M}$ 5HPP-33 for the indicated times (0-24 h), stained with Annexin V-FITC and PI, and analyzed by flow cytometry. Three independent experiments were performed and all gave the same results.

Statistical analysis. Experiments were repeated at least three times, and the probability (P) was calculated by the Student's t-test. $\mathrm{P}$ values $>0.05$ were considered statistically significant.

\section{Results and discussion}

To elucidate the exact anti-myeloma effect of thalidomide and develop new derivatives without causing teratogenic effects, we previously studied the structural development of thalidomide and obtained various analogues with specific molecular properties $(8,9)$. Of these derivatives, 5HPP-33 had the most potent anti-myeloma effects for inhibiting proliferation of RPMI8226, IM9, and U266 cells in time- and dose-dependent manners with $\mathrm{IC}_{50}$ of $1-10 \mu \mathrm{M}$ (Fig. $1 \mathrm{~B}$ and data not shown). In addition, 5HPP-33's activity was more potent than that of a known thalidomide metabolite, 5hydroxythalidomide (data not shown). In contrast to 5HPP-33, thalidomide itself did not inhibit the growth of RPMI8226 
A

\section{Caspase-3 activity}
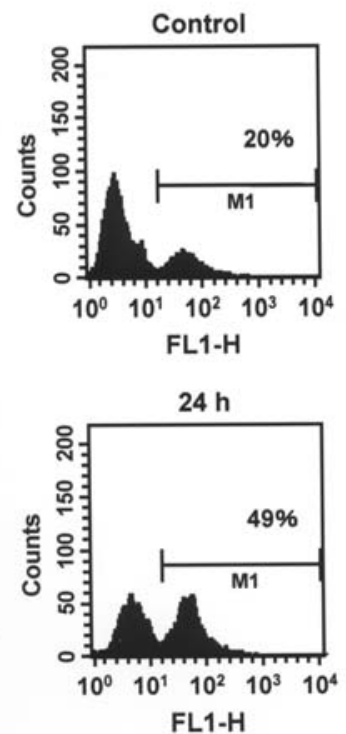
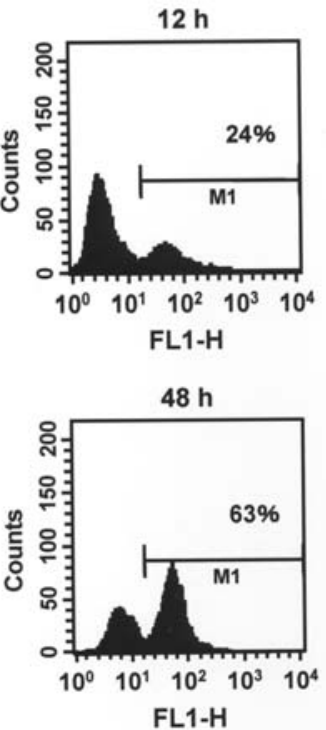

B

\section{Western blotting}

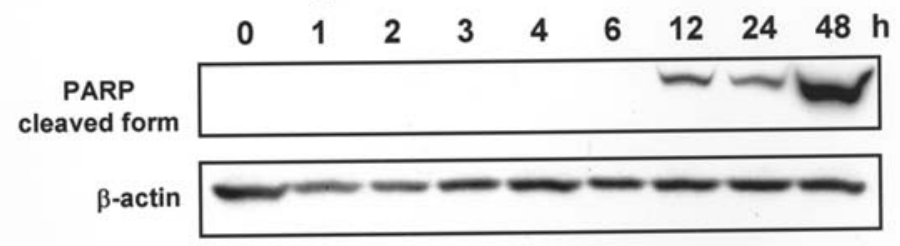

Figure 2. (A) Effects of 5HPP-33 on caspase-3 activity. RPMI8226 cells were treated with $10 \mu \mathrm{M} 5 \mathrm{HPP}-33$ for various times (0-48 h) and analyzed for caspase- 3 activity by flow cytometry. (B) Expression of poly(ADP-ribose) polymerase (PARP) protein in myeloma cells. Cells were treated with $10 \mu \mathrm{M}$ 5 HPP-33 for the indicated times $(0-48 \mathrm{~h})$ and analyzed by Western blotting.

except by exposure to a high concentration $(100 \mu \mathrm{M})$ (Fig. 1B). Cultivation with $10 \mu \mathrm{M}$ 5HPP-33 induced G2/M cell cycle arrest and strongly induced the sub-G1 phase during a 24-h treatment (Fig. 1C). In addition, Annexin V-positive cells increased after the incubation of 5HPP-33 for $12 \mathrm{~h}$ (Fig. 1D). Treatment with 5HPP-33 induced caspase-3 activity and the cleavage of its substrate PARP (Fig. 2A and B). These results indicate that 5HPP-33 led to cell cycle arrest at the G2/M phase followed by apoptosis. From these results, we consider 5HPP-33 to be a potent inhibitor of tubulin polymerization. To address the mechanism underlying the mitotic arrest induced by 5HPP-33, the effects of drug treatment on microtubules were assessed by different approaches. In a tubulin polymerization assay using microtubule protein from porcine brain, 5HPP-33 showed potent tubulin-polymerization-inhibitory activity in a dosedependent manner, with $\mathrm{IC}_{50}$ of $8.1 \mu \mathrm{M}$, comparable to that of the known tubulin polymerization inhibitor, rhizoxin (Fig. 3A). Inhibition of polymerization by 5HPP-33 was completed within 10 min (Fig. 3A and B). For the immunofluorescent study, RPMI8226 cells were grown in chamber slides and exposed to various agents, including 5HPP-33, vincristine, doxorubicin, and paclitaxel before fixation and staining for microtubules with anti-tubulin antibody. In the control cells, microtubules were clearly observed, and individual microtubules often appeared long and relatively straight (Fig. 3C). Paclitaxel treatment seemed to result in large microtubules, which appeared shorter than those of the control. In contrast, 5HPP-33 seemed to inhibit tubulin polymerization in a manner similar to that of vincristine treatment (Fig. 3C). Taken together, these results indicate that 5HPP-33 is a potent inhibitor of tubulin polymerization.
Thalidomide has demonstrated significant activity in both resistant and initial cases of multiple myeloma (3-5). It has been proposed that thalidomide targets a variety of pathways to modulate cell adhesion and inhibit angiogenesis by suppressing production of TNF- $\alpha, \mathrm{IL}-6$, and other cytokines as well as by directly inducing the apoptosis or growth arrest of myeloma cells (12-14). However, the molecular mechanism of the anti-myeloma effect of thalidomide is not clearly understood. Immunomodulatory analogues (IMiDs) of thalidomide have been reported to induce apoptosis in myeloma cells directly (6). However, we showed that thalidomide itself could not directly induce the growth arrest of myeloma cells. It has been reported that thalidomide undergoes spontaneous hydrolytic cleavage to numerous metabolites that are rapidly excreted in urine. It has also been reported that thalidomide is difficult to dissolve in water; therefore, pharmacokinetic data are not well characterized and are highly variable (15). These findings suggest that the anti-myeloma effect of thalidomide might be due to its metabolites, which inhibit growth and induce apoptosis in myeloma cells. Thus, it is important to know which metabolites have the most potent effect in inducing apoptosis of human myeloma cells. We studied structural development of thalidomide and identified a thalidomide analogue, 5HPP-33, with potent in vitro activity against human multiple myeloma cells, which were arrested at G2/M in the mitotic phase. Most anti-mitotic agents perturb microtubules by either inhibiting tubulin polymerization or destabilizing microtubules (16). We demonstrated that 5HPP-33 has the most potent anti-myeloma effects mediated through the inhibition of tubulin polymerization. In addition, we demonstrated that 5HPP-33 inhibits tubulin polymerization 
A

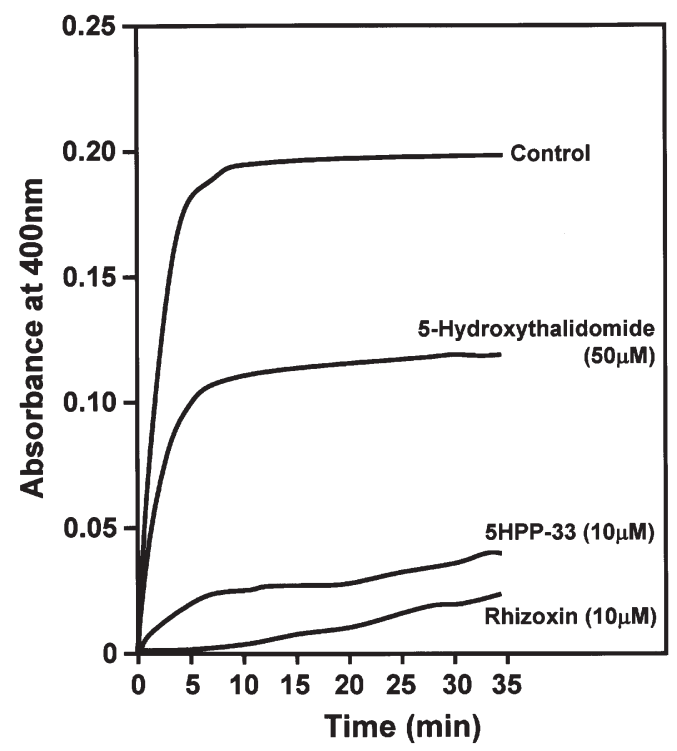

B

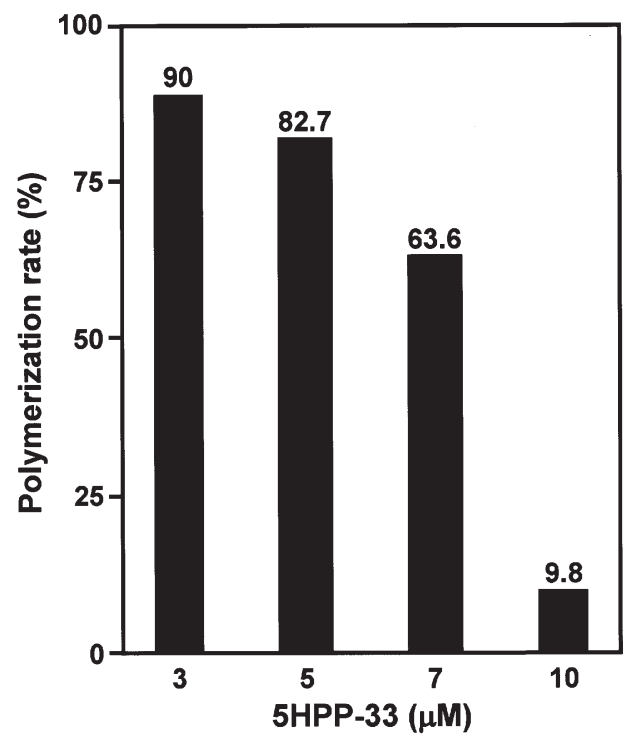

C

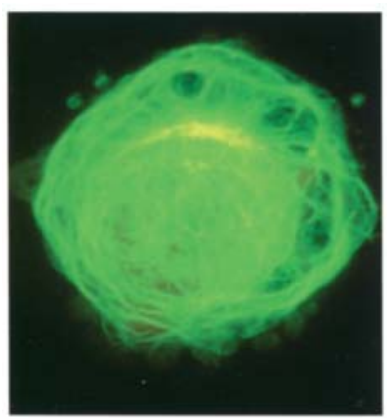

NC

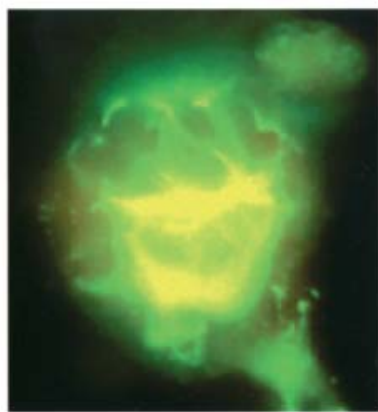

VCR

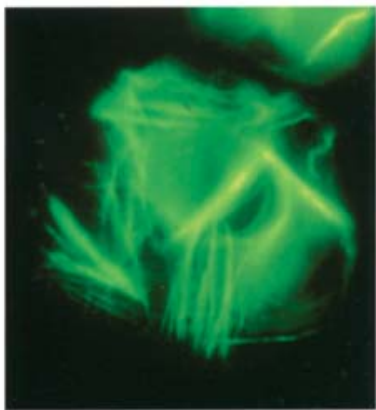

PTX

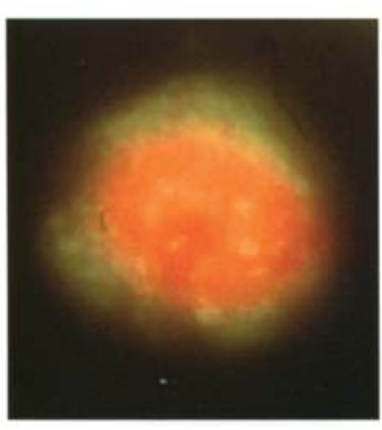

ADR

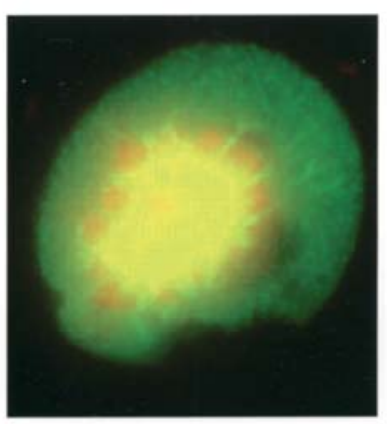

5HPP-33

Figure 3. Tubulin polymerization inhibition assay. (A) Time course curve of the inhibition of tubulin polymerization monitored in terms of turbidity (absorbance at $400 \mathrm{~nm}$ ). A tubulin preparation was incubated at $37^{\circ} \mathrm{C}$ in the presence or absence (control) of compound (thalidomide, 5-hydroxythalidomide, 5HPP-33, and rhizoxin). (B) Polymerization rate after a 30-min incubation with 5HPP-33. 5HPP-33 inhibited polymerization in a dose-dependent manner. The $\mathrm{IC}_{50}$ was proven to be $8.1 \mu \mathrm{M}$. (C) Analysis of $\alpha$-tubulin by confocal microscopy. Analysis of localization of $\alpha$-tubulin in myeloma cells by confocal microscopy after incubation with vincristine (VCR), doxorubicin (ADR), paclitaxel (PTX), and 5HPP-33. Cells were fixed and processed by immunofluorescence labeling with antibody against $\alpha$-tubulin. Staining was analyzed by laser confocal microscopy.

in a manner similar to that of vincristine with myeloma cells. Vinca alkaloids are key drugs for the treatment of multiple myeloma. Taken together, these results suggest that the exact anti-myeloma effect of thalidomide might be due to its metabolite in vivo, which can inhibit tubulin polymerization in myeloma cells.
A variety of thalidomide side effects has been reported, and some of them have often caused the discontinuation of the treatment $(4,17)$. Sedation and constipation are the most frequently noted side effects of thalidomide in clinical settings. Peripheral neuropathy due to axonal degeneration is a well-known complication after prolonged exposure or in 
those with prior neuropathy (18). Venous thromboembolism is uncommon with single-agent thalidomide, but the incidence appears to increase when thalidomide is used in combination with chemotherapeutic agents, in particular anthracyclines $(19,20)$. These numerous side effects of thalidomide might be due to the variety of its metabolites. Therefore, the development of less toxic thalidomide derivatives will be important to reduce the incidence of complications. 5HPP-33 is one of the promising candidates for a new therapeutic agent of multiple myeloma.

In conclusion, our data indicate that 5HPP-33 is a novel inhibitor of tubulin polymerization and that it may be effective in the treatment of multiple myeloma by inducing G2/M phase arrest and apoptosis. Tubulin inhibition might be a direct anti-myeloma mechanism of thalidomide.

\section{Acknowledgements}

This study was supported by The Ministry of Education, Culture, Sports, Science and Technology of Japan, and the Ministry of Health, Welfare and Labour of Japan. We thank Ms. Chika Saito for her excellent technical support.

\section{References}

1. Hideshima T and Anderson KC: Molecular mechanisms of novel therapeutic approaches for multiple myeloma. Nat Rev Cancer 2: 927-937, 2002.

2. Kumar A, Loughran T, Alsina M, Durie BG and Djulbegovic B: Management of multiple myeloma: a systemic review and critical appraisal of published studies. Lancet Oncol 4: 293-304, 2003.

3. Singhal S, Mehta J, Desikan R, Ayers D, Robertson P, Eddlemon P, Munshi N, Anaissie E, Wilson C, Dhodapkar M, Zeldis J, Barlogie B, Siegel D and Crowley J: Anti-tumor activity of thalidomide in refractory multiple myeloma. N Engl J Med 341: 1565-1571, 1999.

4. Weber D: Thalidomide and its derivatives: new promise for multiple myeloma. Cancer Control 10: 375-383, 2003.

5. Cavo M, Zamagni E, Tosi P, Cellini C, Cangini D, Tacchetti P, Testoni N, Tonelli M, de Vivo A, Palareti G, Tura S and Baccarani M: First-line therapy with thalidomide and dexamethasone in preparation for autologous stem cell transplantation for multiple myeloma. Haematologica 89: 826-831, 2004.

6. Hideshima T, Chauhan D, Shima Y, Raje N, Davies F, Tai Y-T, Treon SP, Lin B, Schlossman RL, Richardson P, Muller G, Stirling DI and Anderson KC: Thalidomide and its analogues overcome drug resistance of human multiple myeloma cells to conventional therapy. Blood 96: 2943-2950, 2000.
7. Anderson KC: Lenalidomide and thalidomide: mechanism of action-similarities and differences. Semin Hematol 42: S3-S8, 2005.

8. Miyachi H, Azuma A, Ogasawara A, Uchimura E, Watanabe N, Kobayashi Y, Kato F, Kato M and Hashimoto Y: Novel biological response modifiers: phthalidomides with tumor necrosis factor-alpha production-regulating activity. J Med Chem 40: 2858-2865, 1997.

9. Hashimoto Y: Novel biological response modifiers derived from thalidomide. Curr Med Chem 5: 163-178, 1998.

10. Inatsuki S, Noguchi T, Miyachi H, Oda S, Iguchi T, Kizaki M, Hashimoto $\mathrm{Y}$ and Kobayashi $\mathrm{H}$ : Tubulin-polymerization inhibitors derived from thalidomide. Bioorg Med Chem Lett 15: 321-325, 2005.

11. Yanagawa T, Noguchi $T$, Miyachi $H$, Kobayashi $H$ and Hashimoto Y: Tubulin polymerization inhibitors with a fluorinated phthalidomide skeleton derived from thalidomide. Bioorg Med Chem Lett 16: 4748-4751, 2006.

12. Gupta D, Treon SP, Shima Y, Hideshima T, Podar K, Tai YT, Lin B, Richardson P, Ralph P, Wu L, Payvandi F, Muller G, Stirling DI and Anderson KC: Adherence of multiple myeloma cells to bone marrow stromal cells upregulates vascular endothelial growth factor secretion: therapeutic applications. Leukemia 15: 1950-1961, 2001.

13. Bartlett JB, Dredge K and Dalgleish AG: The evolution of thalidomide and its IMiD derivatives as anticancer agents. Nat Rev Cancer 4: 314-322, 2004.

14. Yasui H, Hideshima T, Richardson PG and Anderson KC: Recent advances in the treatment of multiple myeloma. Curr Pharm Biotechnol 7: 381-393, 2006.

15. Richardson P, Hideshima T and Anderson KC: Thalidomide: emerging role in cancer medicine. Annu Rev Med 53: 629-657, 2002.

16. Jordan MA and Wilson L: Microtubules and actin filaments: dynamic targets for cancer chemotherapy. Curr Opin Cell Biol 10: 123-130, 1998.

17. Kenealy M and Prince HM: Current status of new drugs for the treatment of patients with multiple myeloma. Int Med J 36: 781-789, 2006.

18. Mileshkin L, Stark R, Day B, Seymour JF, Zeldis JB and Prince HM: Development of neuropathy in patients with myeloma treated with thalidomide: patterns of occurrence and the role of electrophysiologic monitoring. J Clin Oncol 24: 4507-4514, 2006.

19. Zangari M, Siegel E, Barlogie B, Anaissie E, Saghafifar F, Fassas A, Morris C, Fink L and Tricot G: Thrombogenic activity of doxorubicin in myeloma patients receiving thalidomide: implications for therapy. Blood 100: 1168-1171, 2002.

20. Osman K, Comenzo R and Rajkumar SV: Deep venous thrombosis and thalidomide therapy for multiple myeloma. $\mathrm{N}$ Engl J Med 344: 1951-1952, 2001. 